\title{
Modeling the stress-strain state of a circular plate subject to general corrosion according to various models
}

\author{
Ilya Ovchinnikov ${ }^{1 *}$ and Vladimir Mavzovin ${ }^{2}$ \\ ${ }^{1}$ Tyumen Industrial University, Russia \\ ${ }^{2}$ Moscow State University of Civil Engineering, Yaroslavskoe shosse, 26, Moscow, 129337, Russia
}

\begin{abstract}
Quite a lot of works have been devoted to the problem of modeling the behavior of thin-walled structures exposed to an aggressive environment leading to corrosive wear of their surface. Researchers have proposed a fairly large set of models of corrosive wear, taking into account the influence of various factors on the kinetics of corrosion (time, material, temperature, the nature of the corrosive environment, the stress-strain state of the structure). Moreover, different authors often propose different models for the same conditions. In the article under consideration, a rather unique comparative study of three corrosion models proposed by different authors (Dolinsky V.M., Gutman E.M., Ovchinnikov I.G.) was carried out to simulate the behavior of the same circular plate subjected to the combined action of load and corrosion wear and tear. Moreover, the identification of the models, that is, the determination of the coefficients included in them, was carried out using the same experimental data. These models were then used to simulate the behavior of plates subject to corrosive wear under various loads. The results of numerical simulation were compared with experimental data obtained during testing of corrosive plates. Interestingly, in the models used, the effect of the stress state on the kinetics of corrosion was taken into account using different invariants of the stress state: the stress intensity in V.M. Dolinsky, medium voltage in the model of E.M. Gutman, and the specific energy in the model of I.G. Ovchinnikov.

The analysis showed that the difference from the experiment when using the three models considered does not exceed 9.3\%. The discrepancy between the results obtained using different models is also within the acceptable range, which suggests that all three models can be used to predict the behavior of plates under corrosive wear conditions. However, it is of interest to conduct research on the predictive capabilities of models on large forecast arms that go beyond the scope of experimental studies. At the same time, carrying out numerical experiments to simulate the behavior of complex structures in a stressed state and subject to corrosive wear, using several models that allow a good description of the experimental data and the most complete consideration of the operating conditions, makes it possible to obtain a more complete and versatile picture of what is happening in design processes, in comparison with the calculations
\end{abstract}

*Corresponding author: bridgeart@mail.ru 
performed according to one model, even if it describes the experimental data well.

\section{Introduction}

Many structures operated under load are exposed to an aggressive environment, as a result of which degradation processes occur in structures, leading to a change in their stress-strain and corrosion state and to a reduction in the period of trouble-free operation. The work of many researchers is devoted to the problem of modeling the behavior of thin-walled metal structures under the combined action of force and thermal fields, as well as aggressive operating environments, a rather detailed review and analysis of which is carried out in monographs [1-4]. The issues of identification and verification of various models of corrosive wear, as well as the problems arising from this, are considered in the monograph [5].

It is clear that to ensure the reliable operation of structures in aggressive environments, one should take into account as many factors as possible that affect the corrosionmechanical behavior of materials and structures made of them. To do this, it is necessary to have and use sufficiently correct models of corrosive wear. It often turns out that different models with different predictive power can be used to describe the same corrosion process. Therefore, the task of comparative analysis of the applicability of various models to the description of the corrosion-mechanical behavior of structures is of both scientific and practical interest.

\section{Problem statement and research method}

In order to carry out a comparative analysis of the applicability of various models of corrosive wear, the identification of which was carried out using the same experimental data, let us consider the problem of modeling the behavior of a circular plate undergoing general corrosion using three models of corrosive wear.

The main system of differential equations of bending of a circular flexible plate is as follows:

$$
\begin{gathered}
D \frac{d}{d r}\left(\Omega^{2} w\right)=\psi+\frac{h}{r} \frac{d \Phi}{d r} \frac{d w}{d r} \\
\frac{d}{d r}\left(\Omega^{2} \Phi\right)=-\frac{\mathrm{E}}{2 r}\left(\frac{d w}{d r}\right)^{2} .
\end{gathered}
$$

Here the operator $\Omega^{2}$ corresponds to the formula:

$$
\Omega^{2} \Phi=\frac{1}{r} \frac{d}{d r}\left(r \frac{d w}{d r}\right),
$$

a $\Psi$ is a function of the load q, determined by the expression:

$$
\psi=\frac{1}{2} \int_{0}^{r} q r d r
$$

In these formulas, $\mathrm{w}$ - is the deflection, $\Phi$ - is the stress function, $\mathrm{E}$ - is the elastic modulus, $r$ - is the radius, $D$ - is the cylindrical stiffness of the plate. 
A plate with a diameter of $\varnothing 150 \mathrm{~mm}$ and a thickness of $4 \mathrm{~mm}$ is considered, made of steel ВСт3. The design diagram of the plate is shown in Figure 1.

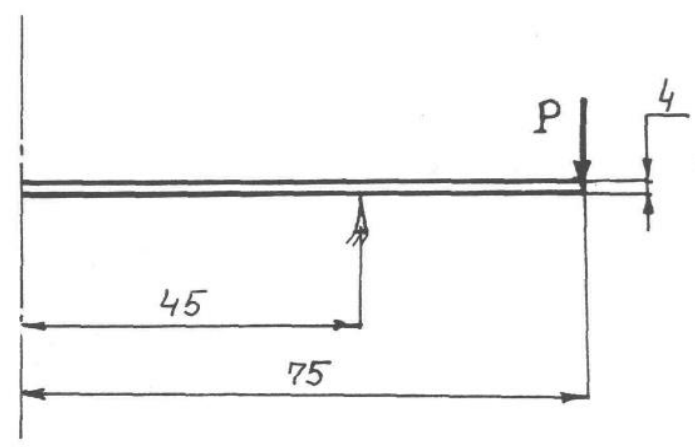

Fig.1. Design scheme of the plate

The plate undergoes general corrosion, the most common models of corrosive wear are used for analysis and their capabilities in predicting the behavior of circular plates are evaluated.

For a comparative study, models were selected that take into account the effect of stresses on the corrosion rate [5]:

model V.M. Dolinsky:

$$
V=V_{0}\left(1+K \sigma_{u}\right)
$$

model E.M. Gutman:

$$
V=V_{0} \cdot \exp \left(\gamma \cdot \sigma_{0}\right)
$$

model I.G. Ovchinnikova:

$$
V=V_{0}(1+\xi \cdot \ni)
$$

In these equations, $\mathrm{V}_{0}$ - is the corrosion rate of the unstressed metal, $\mathrm{K}, \gamma, \xi$ - are the coefficients, $\sigma \mathrm{i}$ is the stress intensity, $\sigma_{0}$ - is the average stress, and $\ni-$ is the specific potential energy.

The coefficients of the models, determined from the same results of experimental studies of round plates, have the following values:

a) for plates of constant loading

$$
V_{0}=0,74 \frac{m m}{y e a r}, K=0,0084 P a^{-1}, \xi=0,15 P a^{-1}, \gamma=0,00815 M P a^{-1}
$$

b) for plates of variable loading

$$
V_{0}=1,113 \frac{m m}{y e a r}, K=0,02 M P a^{-1}, \gamma=0,0138 M P a^{-1}, \xi=0,411 M P a^{-1}
$$


In Fig. 2, 3, 4 show data on the change in the corrosion rate depending on stresses. It follows from the figures that all three considered models describe the experimental results indicated by dots rather satisfactorily.

The error of the V.M. Dolinsky when describing experimental data is $3 \%$, the error of E.M. Gutman - 4\%, the error of the I.G. Ovchinnikov - 12\%. Model coefficients were determined at a base time of 750 hours. As the distance from this point increases, the discrepancies in the results obtained increase.

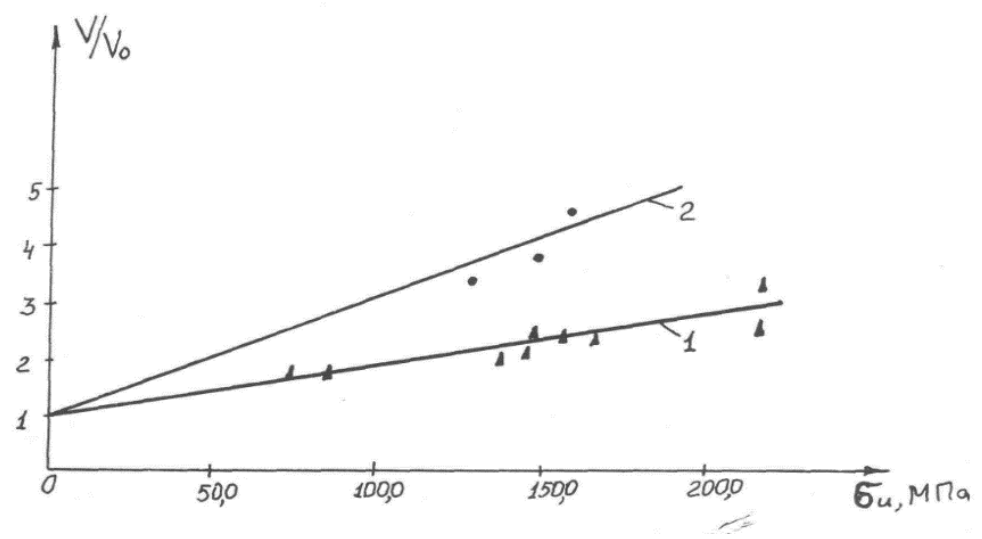

Fig. 2. Dolinsky's model

1 - at constant loading; 2 - under variable loading

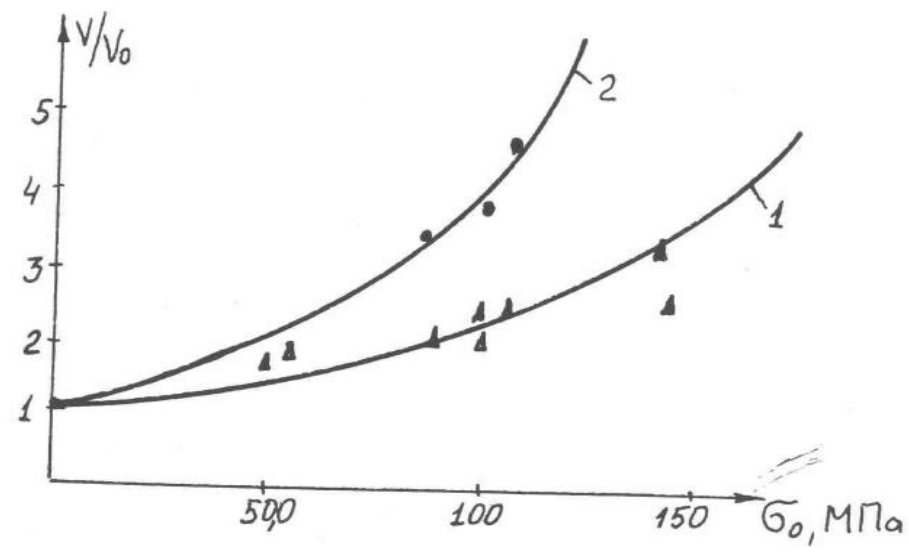

Fig. 3. Gutman's model

1 - at constant loading; 2 - under variable loading 


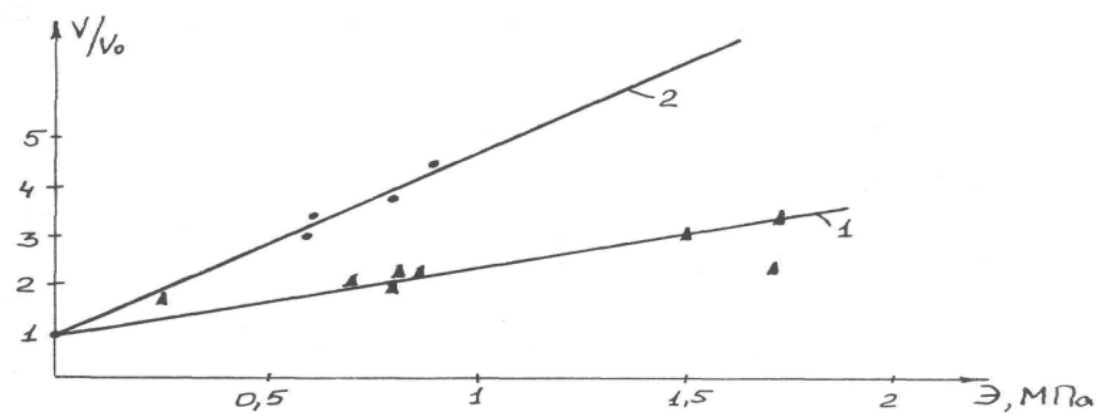

Fig. 4. Ovchinnikov's model

1 - at constant loading; 2 - under variable loading

\section{Results}

For a comparative analysis of the applicability of the three considered models for describing the kinetics of corrosive wear of round plates, we used the results of experimental studies of circular plates [6], in which the deflection of plates subjected to corrosive wear under various loads was determined. The results of calculating the plates, performed using the above three models: 1) V. Dolinsky, 2) E. Gutman and 3) I. Ovchinnikov were compared with experimental data.

Comparison of the data given in Table 1 shows that the discrepancies in the deflections at the center point of the plate to the moment $\tau=750$ hours are on average $4.3 \%$. Moreover, the error of the V. Dolinsky in determining deflections is $6.1 \%$, the error of the E. Gutman $-3.7 \%$, and the error of the I. Ovchinnikov $-2.5 \%$.

Table 1. Comparison of theoretical deflection according to different models with experiment

\begin{tabular}{|c|c|c|c|c|c|}
\hline Load, & Experiment & \multicolumn{3}{|c|}{ Calculated deflections, mm. 10 } \\
\cline { 3 - 5 } & $\mathrm{W}_{\text {exp. }}, \mathrm{mm}$. & \multicolumn{3}{|c|}{} \\
\cline { 3 - 5 } & $10^{2}$ & $\mathrm{~W}_{\text {theor. }}$ & $\begin{array}{c}\text { Dolinsky's } \\
\text { model, W } \\
\text { excluding } \\
\text { corrosion }\end{array}$ & $\begin{array}{c}\text { Gutman's } \\
\text { model, W }\end{array}$ & $\begin{array}{c}\text { Ovchinnikov's } \\
\text { model, W }\end{array}$ \\
\hline
\end{tabular}




\begin{tabular}{|l|l|l|l|l|l|}
\hline 5300 & 22,5 & 24,8 & 23,9 & 23,35 & 23,07 \\
5000 & 21,5 & 23,4 & 22,6 & 22,18 & 21,98 \\
3300 & 15 & 15,4 & 15,35 & 15,27 & 14,99 \\
2880 & 13,5 & 13,5 & 13,8 & 13,23 & 13,57 \\
2750 & 12,4 & 12,9 & 12,75 & 12,48 & 12,56 \\
2630 & 12,4 & 12,3 & 12,17 & 12,06 & 11,99 \\
2500 & 12 & 11,7 & 11,98 & 11,96 & 11,83 \\
\hline
\end{tabular}

Table 2 shows data showing the change in thickness at the center point of the plate, determined using various models and obtained experimentally.

Table 2. Change in the thickness of the corrosive plate according to different models and in experiment.

\begin{tabular}{|c|c|c|c|c|}
\hline \multirow{2}{*}{ load, } & \multicolumn{4}{|c|}{ Change in thickness $\Delta h$, мм } \\
\cline { 2 - 5 } P,H & Experiment & $\begin{array}{c}\text { Dolinsky's } \\
\text { model }\end{array}$ & Gutman's model & $\begin{array}{c}\text { Ovchinnikov's } \\
\text { model }\end{array}$ \\
\hline 5300 & 0,12 & 0,124 & 0,126 & 0,117 \\
10500 & 0,144 & 0,131 & 0,135 & 0,134 \\
14500 & 0,19 & 0,187 & 0,197 & 0,218 \\
\hline
\end{tabular}

The greatest change in thickness by the time $\tau=750 \mathrm{~h}$ is obtained using the model of $\mathrm{I}$. Ovchinnikov. The error in calculating the thickness in the center of the plate according to the model of V. Dolinsky - $9 \%$, according to the model of E. Gutman - $6.6 \%$, according to the model of I. Ovchinnikov $-9.3 \%$.

In fig. 5 shows the data from the study of stresses (experimental data are marked with dots) The lowest voltages are obtained using the V. Dolinsky up to $\tau=180$ hours, and at $\tau$ > 180 hours the lowest stresses are obtained using the E. Gutman. 


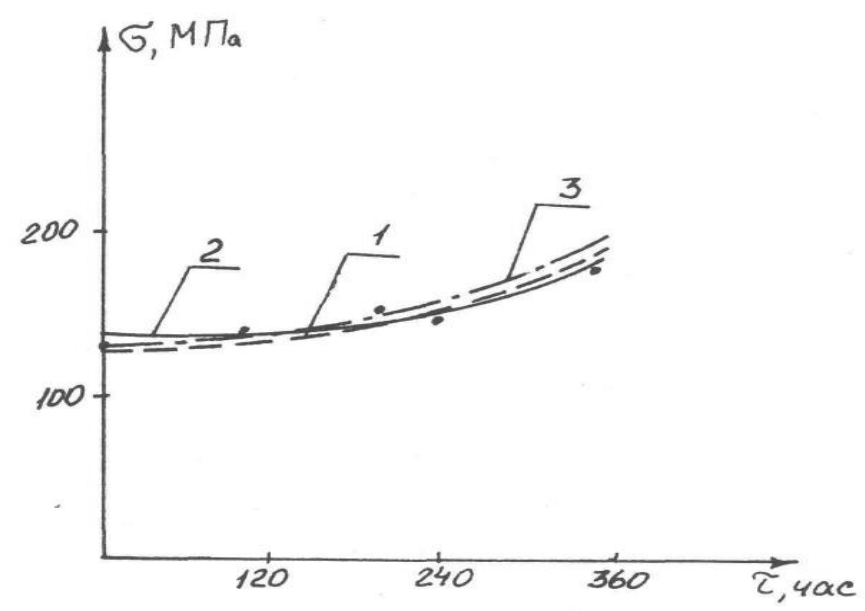

Fig. 5. Changes in stress values in the center of the plate: 1 - calculated using the Dolinsky's model; 2 - Gutman's model; 3 - Ovchinnikov's model

\section{Discussion}

As can be seen, in the considered case, the results of the comparative analysis show that the difference from the experiment when using the three models under consideration does not exceed $9.3 \%$. The discrepancy between the results obtained using different models is also within the acceptable range, which suggests that all three models can be used to predict the behavior of plates under corrosive wear conditions.

\section{Conclusions}

1. The analysis showed that the difference between the results obtained using the considered models of corrosive wear is within the engineering tolerances, and therefore the choice of a specific model for modeling the behavior of plates under corrosion conditions depends on the interests and preferences of the researcher.

2. It is of interest to conduct research on the predictive capabilities of models on large forecast arms that go beyond the scope of experimental studies.

3. At the same time, carrying out numerical experiments using several models, which make it possible to describe well the experimental data and take into account the operating conditions as fully as possible, makes it possible to obtain a more complete and versatile picture of the processes occurring in the structure, in comparison with calculations performed using one model. even if it describes the experimental data well.

\section{Reference}

1. V. Petrov, I. Ovchinnikiv, Yu.Shikhov Calculation of structural elements interacting with an aggressive environment 288 (1987)

2. I. Ovchinnikov Thin-walled structures in conditions of corrosive wear 192 (1995)

3. G. Naumova, I. Ovchinnikov Strength calculations of complex rod and pipeline structures taking into account corrosion damage 227 (2001) 
4. V. Kabanin, G. Naumova, I. Ovchinnikov Strength of shells of revolution subject to corrosive wear in a non-uniform temperature field. 108 (2007)

5. I. Ovchinnikov, I. Ovchinnikov Identification and verification of models of corrosion and deformation processes. 164 (2014)

6. V. Kabanin, O. Kanaeva, I. Ovchinnikov Vestnik VolgGASU 6(21) 87 (2006) 